\begin{tabular}{|c|c|}
\hline Title & V olume-based parameters on FDG PET may predict the proliferative potential of soft-tissue sarcomas \\
\hline Author(s) & $\begin{array}{l}\text { Kitao, Tomoka; Shiga, Tohru; Hirata, Kenji; Sekizawa, Mitsunori; T akei, Toshiki; Y amashiro, Katsushige; T amaki, } \\
\text { Nagara }\end{array}$ \\
\hline Citation & $\begin{array}{l}\text { A nnals of nuclear medicine, 33(1), 22-31 } \\
\text { https://doi.org/10.1007/s12149-018-1298-0 }\end{array}$ \\
\hline Issue Date & $2019-01$ \\
\hline Doc URL & http:/hdl.handle.net/2115/76440 \\
\hline Rights & The final publication is available at link.springer.com \\
\hline Tyре & article (author version) \\
\hline Additional Information & There are other files related to this item in HUSCAP. Check the above URL. \\
\hline File Information & A nnNuclMed33 22.pdf \\
\hline
\end{tabular}

Instructions for use 


\title{
Volume-based parameters on FDG PET may predict the proliferative potential of soft-tissue sarcomas
}

\author{
Tomoka Kitao ${ }^{1,2}$, Tohru Shiga ${ }^{2}$, Kenji Hirata ${ }^{2}$, \\ Mitsunori Sekizawa ${ }^{1}$, Toshiki Takei ${ }^{3}$, Katsushige Yamashiro ${ }^{4}$ and Nagara Tamaki ${ }^{2,5}$ \\ ${ }^{1}$ Radiology Department, Hokkaido Cancer Center, Sapporo, Japan \\ ${ }^{2}$ Department of Nuclear Medicine, Graduate School of Medicine, Hokkaido University, \\ Sapporo, Japan \\ ${ }^{3}$ Department of Diagnostic Radiology, Sapporo City General Hospital, Sapporo, Japan \\ ${ }^{4}$ Department of Pathology, Hokkaido Cancer Center, Sapporo, Japan \\ ${ }^{5}$ Department of Radiology, Graduate School of Medical Science, Kyoto Prefectural \\ University of Medicine, Kyoto, Japan
}

First Author: Tomoka Kitao, MS, Radiology Department, National Hospital Organization Hokkaido Cancer Center, 2-3-54, Kikusui-4, Shiroishi-Ku, Sapporo0030804, Japan. Tel.: +81-11-811-9111; Fax: +81-11-832-0652.

E-mail: tkitao77@yahoo.co.jp

Corresponding Author: Dr. Kenji Hirata, Department of Nuclear Medicine, Graduate School of Medicine, Hokkaido University, Kita 15 Nishi 7, Kita-Ku, Sapporo, Hokkaido 060-8638, Japan. Tel.: +81-11-706-5152; Fax: +81-11-706-7155. E-mail: khirata@med.hokudai.ac.jp

Source of Funding: Funding information is not applicable

Total word count: 4837

Running Title: FDG PET for soft-tissue sarcomas

Type of article: Original article 


\section{ABSTRACT}

Introduction: Soft-tissue sarcomas (STS) are rare types of tumors that have variable levels of tumor differentiation. F-18 fluorodeoxyglucose positron emission tomography (FDG PET) has been established as a useful tool for STS patients, and the metabolic tumor volume (MTV) and total lesion glycolysis (TLG) are reported to be useful in various cancers. We compared the diagnostic value of four PET parameters (maximum standardized uptake value [SUVmax], SUVmean, MTV, and TLG) from two acquisition timings for predicting the expression of the pathological marker of cell proliferation Ki67, based on pathological investigation.

Materials and Methods: In this retrospective study, we investigated 20 patients $(59 \pm 19$ yrs old, 18-87 yrs old) with pathologically confirmed STS who underwent FDG PET before surgical intervention. The patients fasted $\geq 6 \mathrm{hr}$ before the intravenous injection of FDG. The whole body was scanned twice; at an early phase $(61.5 \pm 2.6 \mathrm{~min})$ and at a delayed phase (118.0 \pm 2.1 min) post-injection. The SUVmax, SUVmean, MTV, and TLG of the primary lesion were measured with a tumor boundary determined by SUV $\geq 2.0$. Ki-67 was measured using MIB-1 immunohistochemistry. We used Pearson's correlation coefficient to analyze the relationships between the PET parameters and Ki-67 expressions. The Kaplan-Meier analysis with the log-rank test was performed to compare overall survival between high-group and low-group each of the four PET parameters and Ki-67 expression.

Results: All four PET parameters at each phase showed significant correlations with Ki67. Among them, the Pearson's correlation coefficient (r) was largest for TLG ( $r=0.76$ and 0.77 at the early and delayed phases, respectively), followed by MTV (0.70 and 0.72), SUVmax (r=0.65 and 0.66), and SUVmean ( $r=0.62$ and $r=0.64)$. From early to delayed phases, the SUVmax and SUVmean both increased in all 20 patients, whereas the MTV 
and TLG increased in 13/20 (65\%) and 16/20 (80\%) patients, respectively. None of the \%increases of the PET parameters were significantly correlated with Ki-67. The overall survival was shorter for high-SUVmax, high-SUVmean, high-TLG, and high-Ki67 groups than the other groups, although the difference did not reach statistical significance.

Conclusion: The SUVmax, SUVmean, MTV, and TLG acquired at both 1 and $2 \mathrm{hr}$ after injection showed significant correlations with Ki-67. Among them, correlation coefficient with Ki-67 expression was highest for TLG, although the best parameter should be determined in a larger population. The delayed-phase FDG PET was equally useful as that of early-phase to predict tumor aggressiveness in STS.

\section{Keywords}

soft tissue sarcoma, MTV, TLG, FDG, Ki-67, PET 


\section{Introduction}

Soft-tissue sarcomas (STSs) are rare types of tumors that have various degrees of malignancy. F-18 fluorodeoxyglucose positron emission tomography (FDG PET), a functional imaging modality that reflects glucose metabolism in vivo, has been an essential tool for predicting the malignant potential, staging, prognosis, and treatment response in STS patients [1,2]. Although the use of PET tracers other than FDG such as F-18 fluorothymidine (FLT) [3] and C-11 methionine (MET) has been reported [4], FDG is still the most widely used PET tracer for STS. The standardized uptake value (SUV), which is used as a relative measure of FDG uptake (commonly normalized to the injected dosage and body weight), is used to semi-quantitatively evaluate STSs. In particular, the maximum of the SUV (SUVmax) within the tumor is mostly applied to express the intensity of FDG uptake in the tumor clinically because of its simplicity and high interand intra-observer reproducibility.

Volume-based parameters such as the metabolic tumor volume (MTV) and the total lesion glycolysis (TLG) have been reported to be useful for evaluating various cancers such as lung, head and neck, gynecological cancer, and osteosarcoma [5-9]. These parameters reflect the activity of the glucose metabolism in the entire tumor, whereas the SUVmax reflects only a single voxel (normally, a voxel size $<0.1 \mathrm{ml}$ ).

In STSs, pathological assessments are needed for the detailed diagnosis and staging to determine treatments [10-12]. Tissue specimens from a surgical procedure are normally stained with hematoxylin and eosin (H\&E) to visualize the architecture and cellular features. Immunohistochemical staining techniques are also used to observe the expression of the specific protein in tissues. Ki-67 is a nuclear protein present in proliferating cells, and it is a de facto standard histopathological parameter used as an indicator of cells' proliferative activity. The usefulness of Ki-67 measurement has been 
confirmed, and the grading system using Ki-67 was the strongest prognostic factor [13].

Clinical studies of bone and soft-tissue sarcomas showed that the SUVmax was significantly correlated with the mitotic count [14] and Ki-67 expression [15]. FDG uptake, the SUVmax, and the mean standardized uptake value (SUVmean) were shown to be correlated with Ki-67 in other malignant tumors such as non-small cell lung cancer and breast cancer [16-18]. However, it is not yet established whether the MTV or the TLG is correlated with Ki-67. In addition, the optimal uptake time after FDG injection before image acquisition for the purpose of evaluating proliferative activity has been unclear. To address these questions, we compared four PET parameters (the SUVmax, SUVmean, MTV, and TLG) from two acquisition timings (1 and $2 \mathrm{hr}$ post-injection) in relation to Ki-67 expression assessed by a pathological investigation.

\section{Materials and Methods}

\section{Patients}

In this retrospective study, we investigated a total of 20 patients (10 males, 10 females, age: $59 \pm 19$ years old, range $18-87$ years) who were pathologically confirmed to have STS and underwent FDG PET before any surgical intervention (total resection or biopsy), adjuvant chemotherapy, or radiation therapy at the National Hospital Organization Hokkaido Cancer Center between December 2010 and March 2016. The study population consisted of 17 patients with newly diagnosed STS and 3 with recurrent STS. Of the 20 patients, the tumors of three patients (two newly diagnosed and one recurrent) were diagnosed as low-grade (grade 1) based on Federation National des Centres de Lutte Contre le Cancer (FNCLCC) grading system, which is based on three pathological parameters (tumor differentiation, mitotic count, and tumor necrosis), and the tumors of another 17 patients were diagnosed as high-grade (grade $2, n=7$, five newly 
diagnosed and two recurrent; and grade 3, n=7, seven newly diagnosed; unclassifiable between grades 2 and 3, n=3, three newly diagnosed). The mitotic count information was not available for the unclassifiable patients. The patient histological types are summarized in Table 1. Tumors existed in the head and neck $(n=2)$, the thorax $(n=2)$, the abdomen $(n=7)$, an upper extremity $(n=2)$, or a lower extremity $(n=7)$. The cancer stage was IB $(n=3)$, IIA ( $(n=6)$, IIB ( $n=3)$, III $(n=5)$ or IV $(n=3)$ based on the American Joint Committee on Cancer (AJCC) staging system ver. 7 (2011).

\section{Image acquisition and reconstruction}

An Eminence SET-3000G standalone PET scanner (Shimadzu, Kyoto, Japan) was used for all of the clinical FDG-PET scanning in this patient population. All of the patients fasted for $\geq 6 \mathrm{hr}$ before a single intravenous injection of FDG (218 $\pm 63 \mathrm{MBq}$, range 143$292 \mathrm{MBq}$; or $3.6 \pm 0.5 \mathrm{MBq} / \mathrm{kg}$, range 2.9-4.6 MBq/kg). The blood glucose level was $96 \pm 10 \mathrm{mg} / \mathrm{dl}$. No patient showed a blood glucose level $\geq 150 \mathrm{mg} / \mathrm{dl}$. Each patient's whole body was scanned twice for each study: early imaging at 61.5 $\pm 2.6 \mathrm{~min}$ (range 58-67 min) and delayed imaging at 118 \pm 2.1 min (range 113-122 min) after the FDG injection. The transaxial field of view (FOV) was $512 \mathrm{~mm}$ in diameter. Three-dimensional emission data were acquired in a continuous bed-movement manner $(0.7-0.9 \mathrm{~mm} / \mathrm{sec})$. Transmission scanning was subsequently performed with a ${ }^{137}$ Cs external source for attenuation correction. The transaxial spatial resolution at $1 \mathrm{~cm}$ from the center of the FOV was 3.5 mm at full-width at half maximum.

Images were reconstructed with the dynamic row-action maximum likelihood algorithm (DRAMA), modified from the row-action maximum likelihood algorithm (RAMLA) [19]. The iteration and filter cycle values for DRAMA were 1 and 128, respectively. The image matrix was $128 \times 128$ with the voxel size $4.0 \times 4.0 \times 2.6 \mathrm{~mm}$. A 
post-reconstruction smoothing filter was not applied.

Quantitative image analysis

A total of 40 FDG-PET datasets (early and delayed images from 20 patients) were processed to delineate the tumor. These images were displayed on the monitor in a linear grayscale with a fixed window level of SUV 0-6. The four parameters SUVmax, SUVmean, MTV, and TLG were measured for the primary lesion. The SUV was calculated as [tissue radioactivity concentration $(\mathrm{Bq} / \mathrm{ml})] \times[$ body weight $(\mathrm{g})] /$ [injected radioactivity $(\mathrm{Bq})]$. The MTV was calculated as the tumor volume within the tumor boundary.

The tumor boundary was determined using a fixed threshold of SUV $\geq 2.0$ based on a reported optimal threshold for osteosarcoma [9]. This threshold is relatively low, as SUV $\geq 2.5$ is more often used for lung cancer and head-and-neck cancer. Because STSs occur in extremities or torso fat tissues, the low surrounding background signal levels allow a low SUV threshold to delineate the tumor excluding background activities. The SUVmax and SUVmean within the boundary were measured. The TLG was calculated as MTV $\times$ SUVmean within the same region. For all of the image analyses including the mathematical delineation, and for the parameter calculation, we used Metavol, an opensource software tool that was developed for the efficient measurement of tumor volume on PET-computed tomography (CT) (http://www.metavol.org/) [20].

\section{Pathological assessment}

Pathological data were obtained from CT or ultrasound (US)-guided needle biopsies $(n=4)$, excision biopsies $(n=6)$, and resected specimens of tumors $(n=10)$. These surgical procedures were completed within 3 weeks of the PET scans. The tissues were 
fixed in buffered formalin and embedded in paraffin. The fixed tissues were sectioned, followed by H\&E staining and Ki-67 immunostaining to evaluate cellular proliferation. All pathological diagnoses were made by an experienced pathologist at our institute.

\section{Statistical analysis}

The SUVmax, SUVmean, MTV, and TLG values are expressed as the mean \pm standard deviation (SD). We used paired t-tests to compare these four PET parameters between the early and delayed phases. We used Pearson's correlation coefficient to analyze the relationships between the PET parameters and Ki-67 expressions, and the relationship between the \%increases of the PET parameters and Ki67 expressions. Receiver operating characteristics (ROC) analysis was performed to evaluate PET parameters for distinguishing high-Ki-67 group from low-Ki-67 group. High and low Ki-67 groups were defined by the median as the cut-off value. The KaplanMeier analysis with the log-rank test was performed to compare overall survival (OS) between two groups. OS was defined as the duration from the date of baseline PET scan to death or the date of last contact for surviving patients. The PET parameters and Ki-67 expression were divided into either high group or low group using each median value as the cut-off. P-values $<0.05$ were considered statistically significant. Statistical calculations were carried out using R ver. 3.2.5 (R Project, http://cran.r-project.org).

\section{Results}

The PET parameters at the early and delayed phases

At the early and delayed phases, the SUVmax was $10.2 \pm 6.5$ and $11.9 \pm 7.8$, the SUVmean was $4.2 \pm 1.5$ and $4.5 \pm 1.9$, the MTV was $217 \pm 223 \mathrm{ml}$ and $218 \pm 217 \mathrm{ml}$, and the TLG was $1088 \pm 1471 \mathrm{ml}$ and $1240 \pm 1684 \mathrm{ml}$, respectively. Both the SUVmax and the 
SUVmean increased in the delayed phase in 20/20 (100\%) patients, whereas the MTV and TLG increased in 13/20 (65\%) and 16/20 (80\%) patients. The MTV did not significantly change over the phases, whereas the SUVmax, SUVmean, and TLG significantly increased from the early phase to the delayed phase (Fig. 1).

\section{Correlations between the Ki-67 expression and the PET parameters}

The relationships between Ki-67 expression and the four PET parameters are shown in Figure 2 (early phase) and Figure 3 (delayed phase). Each PET parameter at both phases showed a significant correlation with Ki-67 expression. The Pearson's correlation coefficient was largest for the TLG ( $\mathrm{r}=0.76, \mathrm{p}<0.001$ for the early phase, and 0.77, $\mathrm{p}<0.001$ for the delayed phases), followed by the MTV ( $\mathrm{r}=0.70, \mathrm{p}<0.001$, and 0.72 , $\mathrm{p}<0.001)$, SUVmax $(\mathrm{r}=0.65, \mathrm{p}<0.01$, and 0.66, $\mathrm{p}<0.01)$, and SUVmean $(\mathrm{r}=0.62, \mathrm{p}<0.01$, and 0.64, $\mathrm{p}<0.01$ ). The differences in correlation coefficients between the SUVmax, SUVmean, MTV, and TLG did not reach significance ( $\mathrm{p}=\mathrm{NS})$.

Correlation between the Ki-67 expression and the PET parameters' \%increases

The relationships between the Ki-67 expression and the \%increases of each PET parameter from the early phase to the delayed phase are shown in Figure 4. None of the \%increases of the four parameters was significantly correlated with the Ki-67 expression.

ROC analysis

The ROC curves of the four PET parameters at the early phase and the delayed phase are shown in Figure 5. The area under the curve (AUC) was largest for the TLG ( 0.83 and 0.82 for the early and delayed phases, respectively), followed by the MTV (0.82 
and 0.81), the SUVmean (0.72 and 0.76), and the SUVmax (0.75 and 0.74).

\section{Survival analysis}

The overall survival was shorter for high-SUVmax, high-SUVmean, high-TLG, and high-Ki-67 groups than the other groups, although the difference did not reach statistical significance. (Supplemental Figure 1).

Representative ${ }^{18}$ F-FDG-PET images of three patients are shown in Figure 6. As shown in the cases from the left to the right, the Ki-67 expression, SUVmax, and TLG increased whereas the MTV showed a different tendency.

\section{Discussion}

Our analyses revealed significant correlations between PET parameters and a proliferation-associated pathological marker (Ki-67) in STS. Each of the four PET parameters showed a significant correlation with the expression of Ki-67. Among them, the Pearson's correlation coefficient was largest for the TLG, followed by the MTV, SUVmax, and SUVmean, although the differences of correlation coefficients did not reach statistical significance.

We also evaluated the usefulness of dual-phase FDG PET imaging for STS. The delayed-phase values of TLG, MTV, SUVmax, and SUVmean were also significantly correlated with the Ki-67 expression, and the degree of correction was equivalent between the early and delayed phases. Moreover, none of the \%increases of the early-to-delayed PET parameters was significantly correlated with the Ki-67 expression. We also performed ROC analysis for discriminating high-Ki-67 group from low-Ki-67 group. AUC of MTV and TLG were larger than those of SUVmax and SUVmean at the both phases. Note that each AUC value was equivalent between the early and delayed phases. 
Based on these results, we speculate that delayed PET imaging provides no additional information regarding the aggressiveness of STS. Similarly, there were no significant differences according to the Kaplan-Meier analysis between high-group and low-group by any of the four PET parameters or Ki-67 expression in OS, although we cannot conclude until larger population is studied.

Malignant tumors metabolize glucose dominantly via the glycolytic pathway rather than via the TCA cycle, and these tumors thus consume more glucose than normal cells do to produce the same amount of adenosine triphosphate (ATP), which is known as the Warburg effect [21]. In addition, malignant cells may need more ATP to accelerate cell proliferation, which may also increase glucose consumption. In the present study, both the MTV and the TLG were strongly correlated with the Ki-67 expression. One possible reason is that more-rapidly growing tumors may be relatively larger at onset. The SUVmax was also correlated with Ki-67, which was consistent with the report from Watanabe et al. showing that the Ki-67 expression was higher in high-SUVmax sites in non-Hodgkin lymphoma [22]. Theoretically, SUVmax is not the best indicator for at least 2 reasons. First, Ki-67 was not always evaluated at the same site that shows SUVmax. Ki-67 staining of a great number of slices from an entire tumor is not practical, and, in biopsy cases, the highest-FDG-uptake part is not always sampled. Second, many of the tumors were large ( $>5 \mathrm{~cm}$, stage IB, IIB and above) and had strong heterogeneity with micro-necrosis causing underestimation of the maximum concentration of FDG due to partial volume effect. These factors should have weakened the correlation between the SUVmax and Ki-67 expression.

In this context, we investigated the MTV and the TLG because they may have potential to overcome the above shortcomings of SUVmax. In literature, the relationship between Ki-67 and prognostic factors has been reported from several clinical studies of 
other malignant tumors [9,23-28]. Byun et al. showed that the MTV can more accurately predict the clinical outcomes of patients with osteosarcoma compared to the SUVmax and TLG on PET [9]. Park et al. showed that the TLG is a better predictive factor than the SUVmax and MTV for the outcomes of patients with NSCLC [28].

In the current study, we observed that both the MTV and the TLG showed larger correlation coefficients than SUVmax, although the difference was not demonstrated statistically. In particular, TLG showed a stronger correlation with Ki-67 than the MTV. One of the reasons is that MTV may not fully reflect the metabolic activity of the tumor. For instance, MTV does not discriminate a voxel full of active cells (e.g., SUV=10) from a voxel with micro-necrotic foci coexisting with active cells (e.g., SUV=3), because these voxels are considered equivalent in the MTV concept. In contrast, TLG can reflect the above difference. Based on the definitions,

$$
\begin{gathered}
M T V=n v_{0} \\
S U V_{\text {mean }}=\frac{1}{n} \sum_{i}^{n} S U V_{i}
\end{gathered}
$$

where $n$ is the number of voxels within the VOI, $v_{0}$ is the volume of the single voxel, and $\mathrm{SUV}_{\mathrm{i}}$ is the SUV of the $i$ th voxel $(i=1,2,3, \ldots, n)$ within the tumor boundary. Thus, TLG can be expressed as follows:

$$
T L G=M T V \times S U V_{\text {mean }}=n v_{0} \times \frac{1}{n} \sum_{i}^{n} S U V_{i}=v_{0} \sum_{i}^{n} S U V_{i}
$$

The equation indicates that TLG is proportional to the sum of SUV of each voxel within the tumor boundary. Thus, TLG can overcome the problem regarding MTV mentioned above. In addition, SUVmax is underestimated when the area of the highest uptake is smaller than the spatial resolution of the scanner, while TLG can count the spilled-over activity into the surrounding voxels. Therefore, we suggest that TLG may theoretically 
most appropriately indicate the malignant potential of the tumor.

Before using volume-based parameters in clinical settings, we should be aware that the MTV and TLG can be affected by differences in PET scanners, the uptake time, and delineation methods $[29,30]$. Harmonization is needed to use these parameters in multicenter clinical trials [31].

We sought to clarify the usefulness of dual-phase imaging for predicting cell proliferating activities in this retrospective study. Other groups reported that dual-phase imaging was valuable to differentiate between benign and malignant lesions, and the SUVmax increment of the lung cancer could be a prognostic factor [32,33]. In visual assessment, it is well known that dual-phase imaging is also useful to distinguish malignant lymph nodes' uptake from physiological FDG uptake in normal bowels. However, in the present study, the measurements derived from the dual-phase images did not improve the correlation coefficient between the PET parameters and the Ki-67 index. From the early phase to the delayed phase, the SUVmax, SUVmean, and TLG increased significantly, whereas the MTV did not change significantly. Therefore, the dual-phase imaging in this study did not provide additional value compared to single-phase imaging in terms of grading the malignancies. We observed previously that the MTV calculated with a threshold of SUV 2.0 or 2.5 was not affected by the uptake time, whereas higher thresholds ( $\geq 3.0$ ) produced MTV values that depended on the uptake time [29]. In addition, the SUVmax and TLG were influenced by the uptake time.

The clinical importance of our present findings is that they can be used to help surgeons predict the pathological aggressiveness of STSs before the operation, and the surgeons can thus modify the surgical procedure (biopsy or resection) based on the information obtained from PET. As a possible treatment strategy, if a tumor shows a large TLG value, the surgeon may plan a more extensive procedure with a larger margin and 
more extensive lymph node dissection, and vice versa.

This study has several limitations. First, as this was a retrospective study and included only a small number of patients who underwent surgical intervention, selection bias could have affected the results. Second, the PET scanner used in this study is not a state-of-art PET-CT scanner. Current scanners with higher spatial resolution may generate better images to provide better predictive factors. Third, we calculated the MTV and TLG, but recent studies often use texture parameters that reflect the heterogeneity of tumor metabolism, based on a hypothesis that the more aggressive tumors may tend to be more heterogeneous in metabolism. Further studies are necessary to investigate whether texture analysis provides value additional to that shown by the MTV and TLG in soft-tissue sarcomas.

\section{Conclusions}

The SUVmax, SUVmean, MTV, and TLG acquired at both 1 and 2 hr after FDG injection showed significant correlations with the expression of the proliferation marker of Ki-67 in soft-tissue sarcomas. Among them, correlation coefficient with Ki-67 expression was highest for TLG, although the best parameter should be determined in a larger population. The delayed-phase FDG PET was equally useful as that of early-phase to predict Ki-67 expression. 


\section{The list of abbreviations}

CT: computed tomography

DRAMA: dynamic row-action maximum likelihood algorithm

FDG: fluorodeoxyglucose

FLT: fluorothymidine

FNCLCC: Federation National des Centres de Lutte Contre le Cancer

FOV: field of view

H\&E: hematoxylin and eosin

MET: methionine

MTV: metabolic tumor volume

OS: overall survival

PET: positron emission tomography

RAMLA: row-action maximum likelihood algorithm

STS: Soft-tissue sarcomas

SUV: standardized uptake value

SUVmax: maximum of SUV

SUVmean: mean of SUV

TLG: total lesion glycolysis

US: ultrasound

\section{Acknowledgements}

We would like to thank Eriko Suzuki for her great support in the preparation of this manuscript. We would also like to thank the members of the department of Nuclear Medicine, Hokkaido University for their hospitality during the research. 


\section{Funding}

Funding information is not applicable.

\section{Availability of data and materials}

The datasets supporting the conclusions of this article are included within the article.

\section{Authors' contributions}

Design of the study: TK KH

Investigation: TK TS KH NT

Formal analysis: TK KY.

Software: KH.

Supervision: TS MS TT KY NT.

Writing - original draft: TK KH.

Writing - review \& editing: TK TS KH MS TT KY NT.

\section{Competing interests}

The authors declare that they have no competing interests.

\section{Consent to publish}

Not applicable.

\section{Ethics approval and consent to participate}

This retrospective study was approved by the institutional ethics committee of Hokkaido Cancer Center. The informed consent was waived from individual participants in the retrospective study according to the institutional ethics committee of Hokkaido 
Cancer Center (\#27-43). Patient records/information was anonymized and de-identified prior to analysis.

\section{Statement of human rights}

All procedures performed in studies involving human participants were in accordance with the ethical standards of the institutional and/or national research committee and with the 1964 Helsinki declaration and its later amendments or comparable ethical standards. 


\section{References}

1. Ha SC, Oh JS, Roh J-L, Moon H, Kim JS, Cho K-J, et al. Pretreatment tumor SUVmax predicts disease-specific and overall survival in patients with head and neck soft tissue sarcoma. Eur J Nucl Med Mol Imaging. 2017;44:33-40.

2. Liu C-YC-L, Yen C-C, Chen W-M, Chen T-H, Chen PC-H, Wu H-TH, et al. Soft tissue sarcoma of extremities: the prognostic significance of adequate surgical margins in primary operation and reoperation after recurrence. Ann Surg Oncol. 2010;17:2102-11.

3. Been LB, Suurmeijer AJH, Elsinga PH, Jager PL, van Ginkel RJ, Hoekstra HJ. 18F-Fluorodeoxythymidine PET for evaluating the response to hyperthermic isolated limb perfusion for locally advanced soft-tissue sarcomas. J Nucl Med. 2007;48:367-72.

4. Ghigi G, Micera R, Maffione AM, Castellucci P, Cammelli S, Ammendolia I, et al. ${ }^{11} \mathrm{C}$-methione vs. ${ }^{18} \mathrm{~F}$-FDG PET in soft tissue sarcoma patients treated with neoadjuvant therapy: Preliminary results. In Vivo. 2009 Jan-Feb;23(1):105-10.

5. Kao CH, Lin SC, Hsieh TC, Yen KY, Yang SN, Wang YC, et al. Use of pretreatment metabolic tumour volumes to predict the outcome of pharyngeal cancer treated by definitive radiotherapy. Eur J Nucl Med Mol Imaging. 2012;39:1297-305

6. Lim R, Eaton A, Lee NY, Setton J, Ohri N, Rao S, et al. ${ }^{18}$ F-FDG PET/CT metabolic tumor volume and total lesion glycolysis predict outcome in oropharyngeal squamous cell carcinoma. J Nucl Med. 2012;53:1506-13.

7. Lee JW, Cho A, Lee JH, Yun M, Lee JD, Kim YT, et al. The role of metabolic tumor volume and total lesion glycolysis on ${ }^{18} \mathrm{~F}$-FDG PET/CT in the prognosis of epithelial ovarian cancer. Eur J Nucl Med Mol Imaging. 2014;41:1898-906.

8. Im H-J, Pak K, Cheon GJ, Kang KW, Kim S-J, Kim I-J, et al. Prognostic value of volumetric parameters of ${ }^{18}$ F-FDG PET in non-small-cell lung cancer: a metaanalysis. Eur J Nucl Med Mol Imaging. 2014;42:241-51.

9. Byun BH, Kong C-B, Park J, Seo Y, Lim I, Choi CW, et al. Initial metabolic tumor volume measured by ${ }^{18} \mathrm{~F}$-FDG PET/CT can predict the outcome of osteosarcoma of the extremities. J Nucl Med. 2013;54:1725-32.

10. Guillou L, Coindre JM, Bonichon F, Nguyen BB, Terrier P, Collin F, et al. Comparative study of the National Cancer Institute and French Federation of Cancer Centers Sarcoma Group grading systems in a population of 410 adult patients with soft tissue sarcoma. J Clin Oncol. 1997;15:350-62.

11. Pisters PW, Leung DH, Woodruff J, Shi W, Brennan MF. Analysis of prognostic factors in 1,041 patients with localized soft tissue sarcomas of the extremities. $\mathrm{J}$ 
Clin Oncol. 1996;14:1679-89.

12. Neuville A, Chibon F, Coindre J-M. Grading of soft tissue sarcomas: From histological to molecular assessment. Pathology. 2014;46:113-20.

13. Hasegawa T, Yokoyama R, Lee YH, Shimoda T, Beppu Y, Hirohashi S. et al. Prognostic relevance of a histological grading system using MIB-1 for adult softtissue sarcoma. Oncology. 2000;58: 66-74.

14. Rakheja R, Makis W, Skamene S, Nahal A, Brimo F, Azoulay L, et al. Correlating metabolic activity on ${ }^{18} \mathrm{~F}$-FDG PET/CT with histopathologic characteristics of osseous and soft-tissue sarcomas: A retrospective review of 136 patients. Am J Roentgenol. 2012;198: 1409-16.

15. Walter F, Federman N, Apichairuk W, Nelson S, Phelps ME, Allen-Auerbach M, et al. ${ }^{18} \mathrm{~F}$-fluorodeoxyglucose uptake of bone and soft tissue sarcomas in pediatric patients. Pediatr Hematol Oncol. 2011;28:579-87.

16. Yamamoto Y, Nishiyama Y, Ishikawa S, Nakano J, Chang SS, Bandoh S, et al. Correlation of ${ }^{18}$ F-FLT and ${ }^{18}$ F-FDG uptake on PET with Ki-67 immunohistochemistry in non-small cell lung cancer. Eur J Nucl Med Mol Imaging. 2007;34:1610-16.

17. Buck AK, Halter G, Schirrmeister H, Kotzerke J, Wurziger I, Glatting G, et al. Imaging proliferation in lung tumors with PET: ${ }^{18} \mathrm{~F}-\mathrm{FLT}$ versus ${ }^{18} \mathrm{~F}$-FDG. J Nucl Med. 2003;44:1426-31.

18. Avril N, Menzel M, Dose J, Schelling M, Weber W, Jänicke F, et al. Glucose metabolism of breast cancer assessed by ${ }^{18} \mathrm{~F}-\mathrm{FDG}$ PET: Histologic and immunohistochemical tissue analysis. J Nucl Med. 2001;42:9-16.

19. Tanaka E, Kudo H. Subset-dependent relaxation in block-iterative algorithms for image reconstruction in emission tomography. Phys Med Biol. 2003;48:1405-22.

20. Hirata K, Kobayashi K, Wong KP, Manabe O, Surmak A, Tamaki N, et al. A semi-automated technique determining the liver standardized uptake value reference for tumor delineation in FDG PET-CT. PLoS One. 2014;9:e105682.

21. Vander Heiden MG, Cantley LC, Thompson CB. Understanding the Warburg effect: The metabolic requirements of cell proliferation. Science. 2009;324:102933.

22. Watanabe R, Tomita N, Takeuchi K, Sakata S, Tateishi U, Tanaka M, et al. SUVmax in FDG-PET at the biopsy site correlates with the proliferation potential of tumor cells in non-Hodgkin lymphoma. Leuk Lymphoma. 2010;51:279-83.

23. Liao S, Penney BC, Wroblewski K, Zhang H, Simon CA, Kampalath R, et al. Prognostic value of metabolic tumor burden on ${ }^{18} \mathrm{~F}$-FDG PET in nonsurgical patients with non-small cell lung cancer. Eur J Nucl Med Mol Imaging. 
2012;39:27-38.

24. Liu J, Dong M, Sun X, Li W, Xing L, Yu J. Prognostic value of ${ }^{18}$ F-FDG PET/CT in surgical non-small cell lung cancer: A meta-analysis. PLoS One. 2016;11:e0146195.

25. Satoh Y, Nambu A, Ichikawa T, Onishi H. Whole-body total lesion glycolysis measured on fluorodeoxyglucose positron emission tomography/computed tomography as a prognostic variable in metastatic breast cancer. BMC Cancer. 2014;14:525.

26. Kim JW, Oh JS, Roh J-L, Kim JS, Choi S-H, Nam SY, et al. Prognostic significance of standardized uptake value and metabolic tumour volume on ${ }^{18} \mathrm{~F}$ FDG PET/CT in oropharyngeal squamous cell carcinoma. Eur J Nucl Med Mol Imaging. 2015;42:1353-61.

27. Ryu IS, Kim JS, Roh J-L, Lee JH, Cho K-J, Choi S-H, et al. Prognostic value of preoperative metabolic tumor volume and total lesion glycolysis measured by ${ }^{18}$ F-FDG PET/CT in salivary gland carcinomas. J Nucl Med. 2013;54:1032-8.

28. Park SY, Cho A, Yu WS, Lee CY, Lee JG, Kim DJ, et al. Prognostic value of total lesion glycolysis by ${ }^{18} \mathrm{~F}-\mathrm{FDG} \mathrm{PET} / \mathrm{CT}$ in surgically resected stage IA nonsmall cell lung cancer. J Nucl Med. 2015;56:45-9.

29. Kitao T, Hirata K, Shima K, Hayashi T, Sekizawa M, Takei T, et al. Reproducibility and uptake time dependency of volume-based parameters on FDG-PET for lung cancer. BMC Cancer. 2016;16:576.

30. Liu H, Chen P, Wroblewski K, Hou P, Zhang C-P, Jiang Y, et al. Consistency of metabolic tumor volume of non-small-cell lung cancer primary tumor measured using ${ }^{18}$ F-FDG PET/CT at two different tracer uptake times. Nucl Med Commun. 2016;37:50-6.

31. Wahl RL, Jacene H, Kasamon Y, Lodge MA. From RECIST to PERCIST: Evolving considerations for PET response criteria in solid tumors. J Nucl Med. 2009;50:122S-50S.

32. Houseni M, Chamroonrat W, Zhuang J, Gopal R, Alavi A, Zhuang H. Prognostic implication of dual-phase PET in adenocarcinoma of the lung. J Nucl Med. 2010;51:535-42.

33. Chen HHW, Lee B-F, Su W-C, Lai Y-H, Chen H-Y, Guo H-R, et al. The increment in standardized uptake value determined using dual-phase ${ }^{18} \mathrm{~F}$-FDG PET is a promising prognostic factor in non-small-cell lung cancer. Eur J Nucl Med Mol Imaging. 2013;40:1478-85. 


\section{Figure Legends}

Fig. 1. The MTV (c) did not significantly change, whereas the SUVmax (a), SUVmean (b) and TLG (d) increased significantly from the early phase to the delayed phase.

Fig. 2. Scatter plots of PET parameters at the early phase versus the Ki-67 expression. The SUVmax (a), SUVmean (b), MTV (c) and TLG (d) showed significant correlations with Ki-67 expression, and among them, the Pearson's correlation coefficient was largest for the TLG. The Pearson's correlation coefficients did not change at the delayed phase.

Fig. 3. Scatter plots of the PET parameters at the delayed phase versus the Ki-67 expression. Similarly to the early phase (Fig. 2), the SUVmax (a), SUVmean (b), MTV (c) and TLG (d) showed significant correlations with Ki-67 expression. The Pearson's correlation coefficient was largest for the TLG.

Fig. 4. Scatter plots of the \%increases of the SUVmax (a), SUVmean b), MTV (c) and TLG (d) versus the Ki-67 expression. The \%increases of all PET parameters were not significantly correlated with Ki-67.

Fig. 5. ROC curves for distinguishing high-Ki-67 group from low-Ki-67 group by PET parameters at the early phase (a) and the delayed phase (b).

Fig. 6. ${ }^{18}$ F-FDG-PET images (early phase) of three representative patients with softtissue sarcomas. (a) Synovial sarcoma image with relatively low Ki-67 expression. (b) Pleomorphic undifferentiated sarcoma image with moderate Ki-67 expression. (c) Dedifferentiated liposarcoma image with high Ki-67 expression. Among them, the Ki- 
67 expression, SUVmax, and TLG values showed the same tendency, whereas the MTV did not. 
Table 1. Patients and tumor characteristics

Patient age $\quad 59 \pm 19$ (range 18-87)

Patient sex:

Male

Female

10

Tumor location:

Head and neck 2

Thorax 2

Abdomen $\quad 7$

Upper extremity 2

Lower extremity 7

Tumor grade:

FNCLCC grade1 3

FNCLCC grade2 7

FNCLCC grade3 7

Ungradable (between grade 2 and 3) 3

AJCC stage:

I B 3

$\begin{array}{ll}\text { II A } & 6\end{array}$

II B

III

IV 3

Tumor histology:

Leiomyosarcoma 3

Liposarcoma 3

Rhabdomyosarcoma 1

Myxofibrosarcoma

Synovial sarcoma $\quad 1$

Extraskeletal Ewing's sarcoma

MPNST 3

Undifferentiated 7

AJCC: American Joint Committee on Cancer, FNCLCC: Federation National des Centres de Lutte Contre le Cancer; MPNST: malignant peripheral nerve sheath tumor. 
Fig. 1

(a)

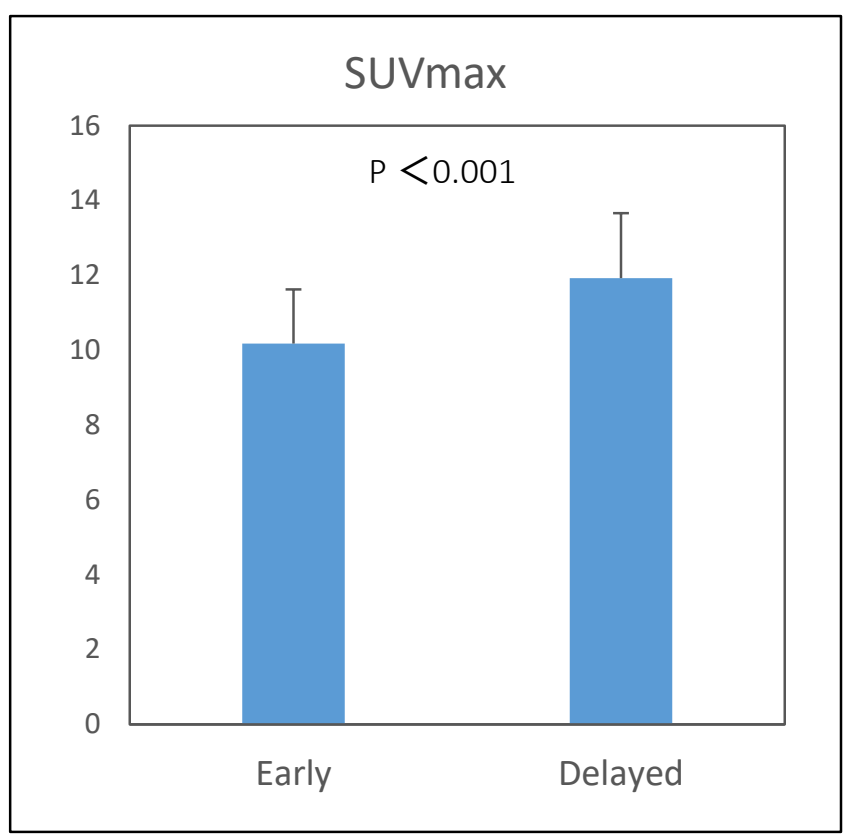

(c)

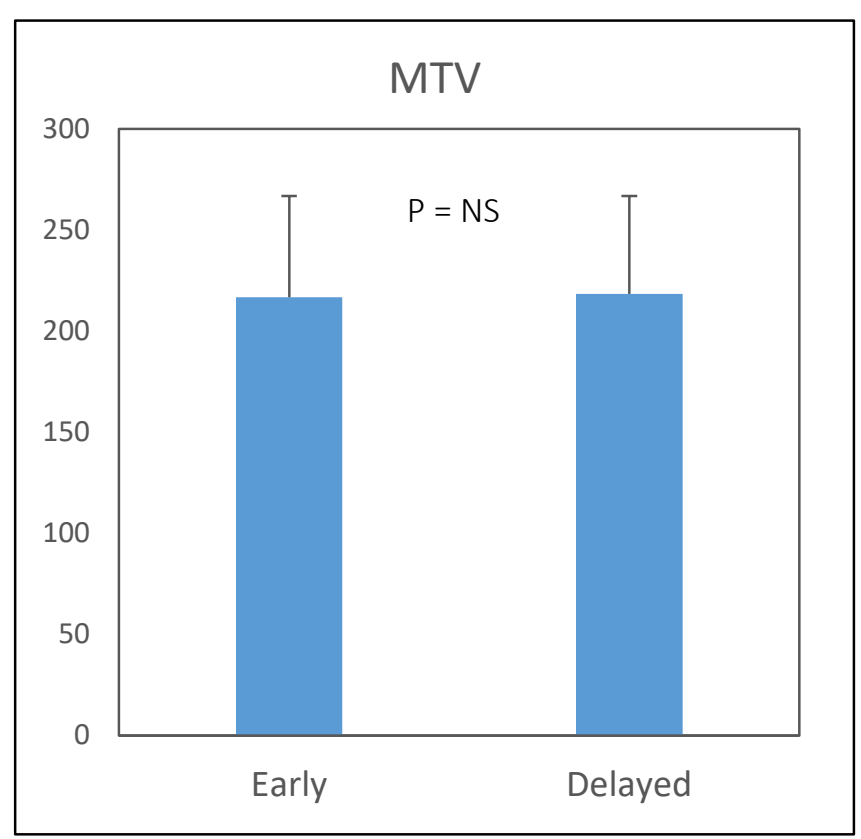

(b)

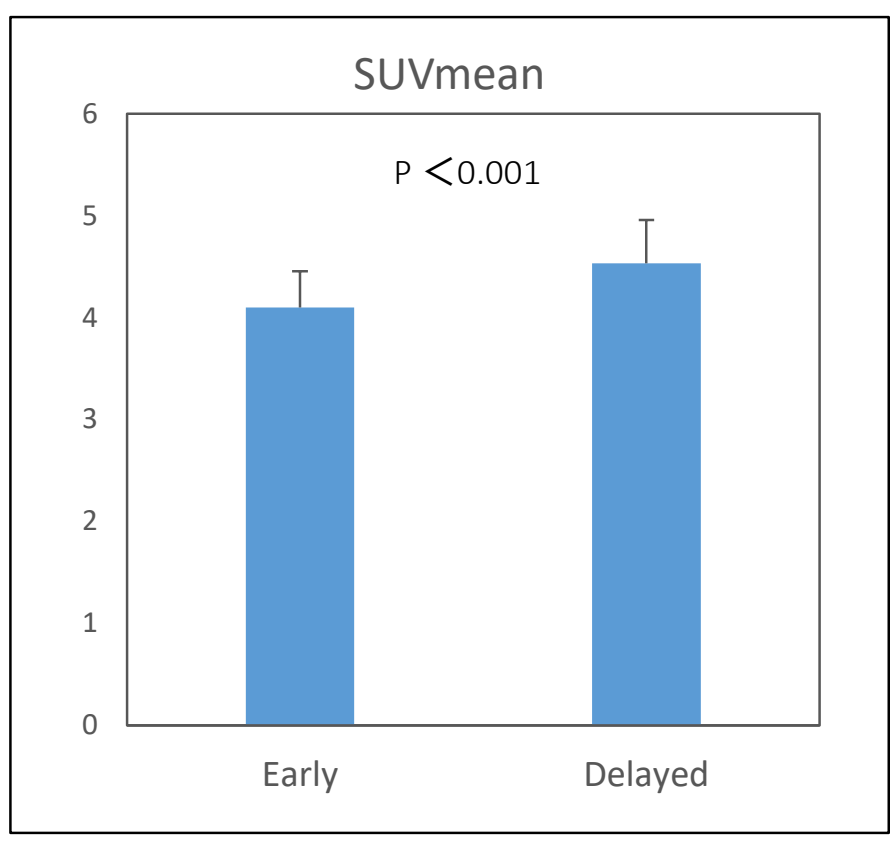

(d)

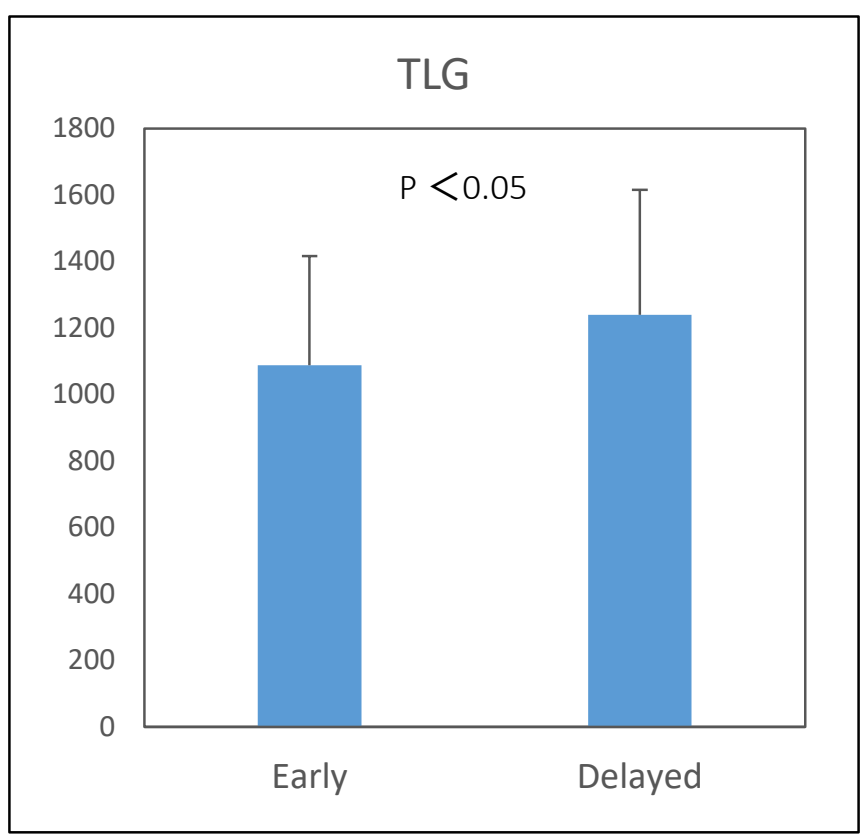

P-value by paired t-test 
Fig. 2

(a)

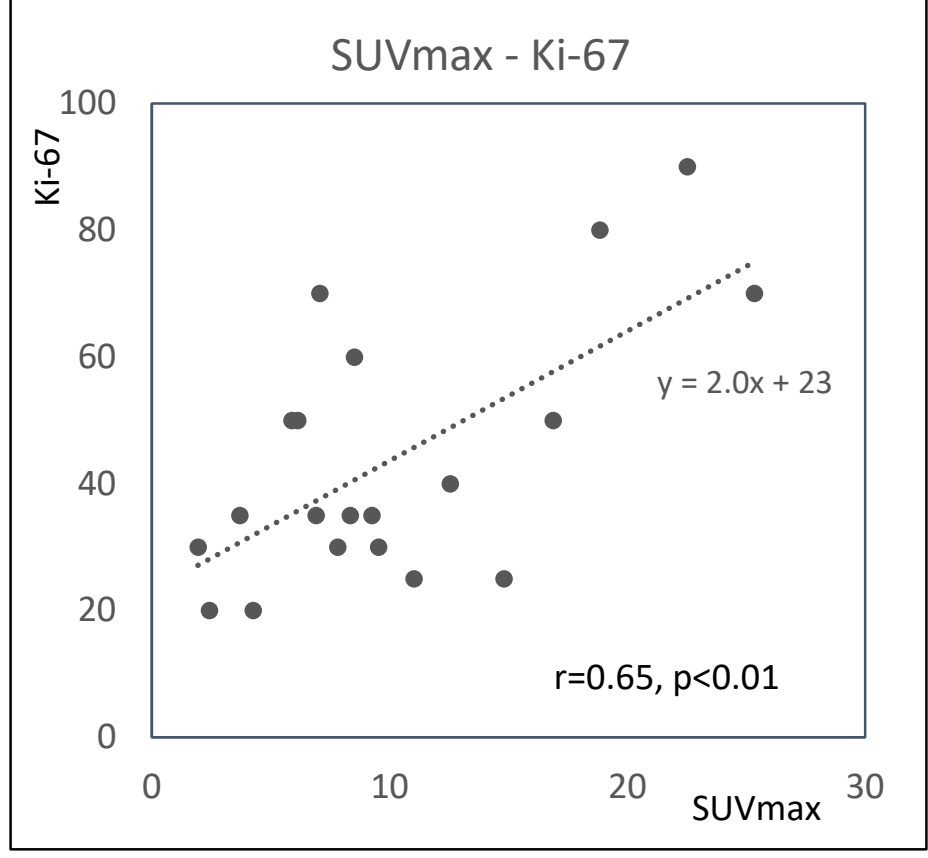

(c)

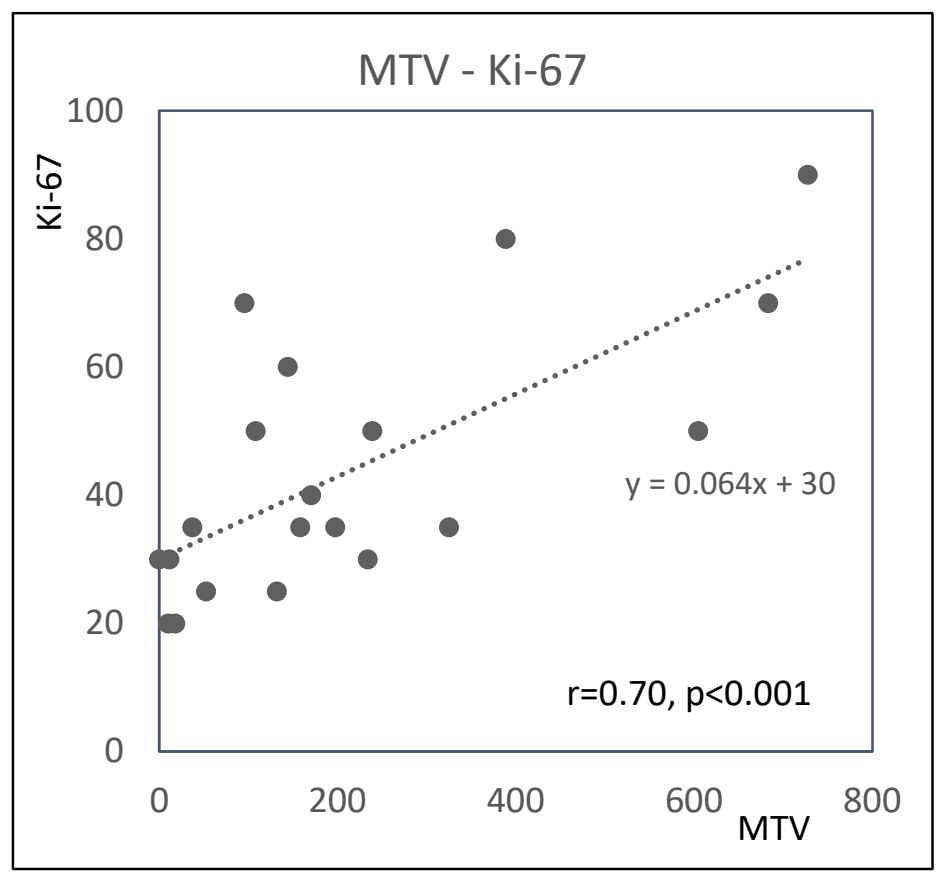

(b)

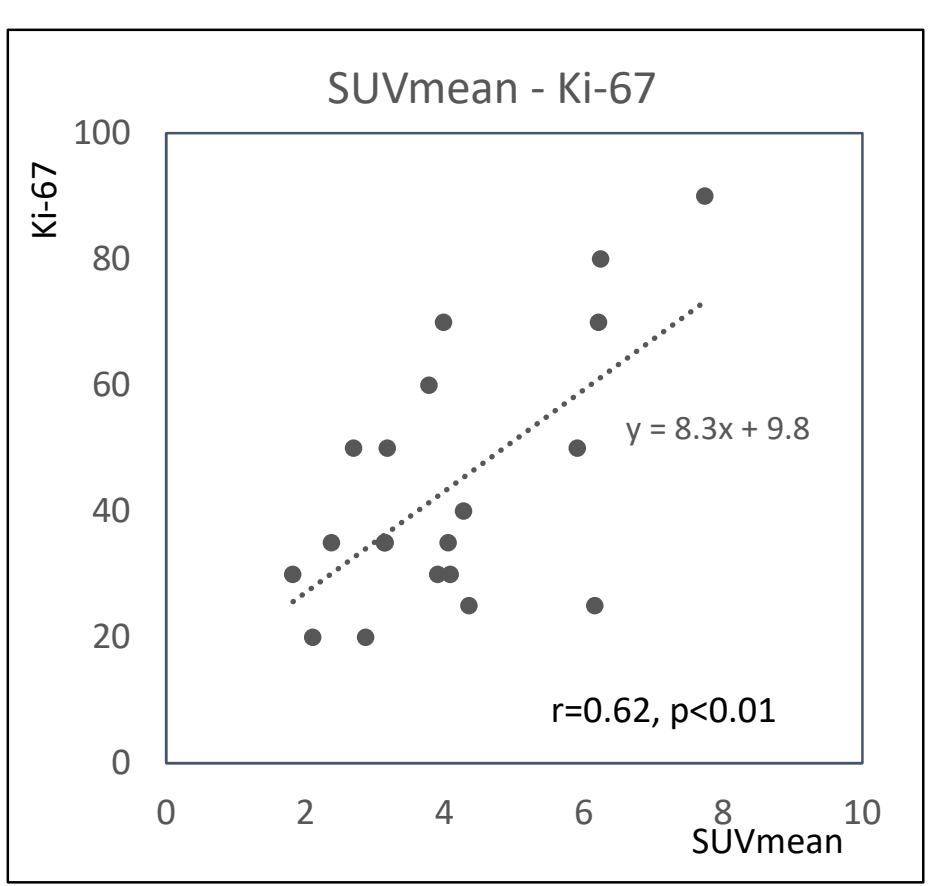

(d)

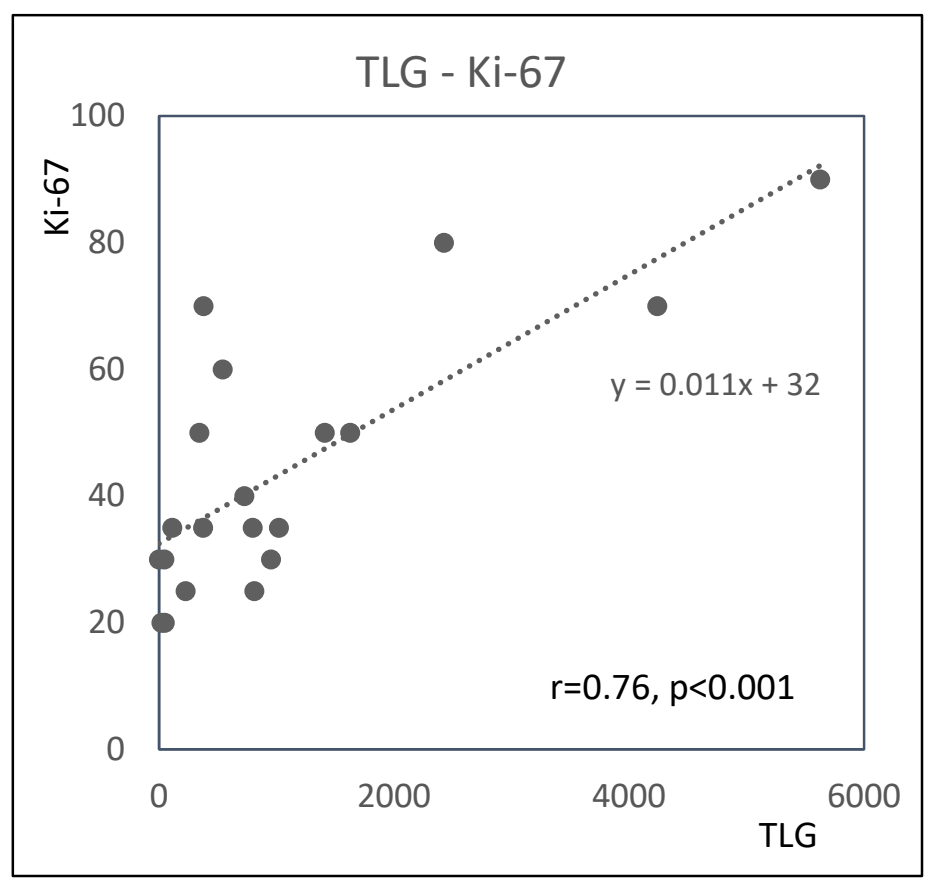


Fig. 3

(a)

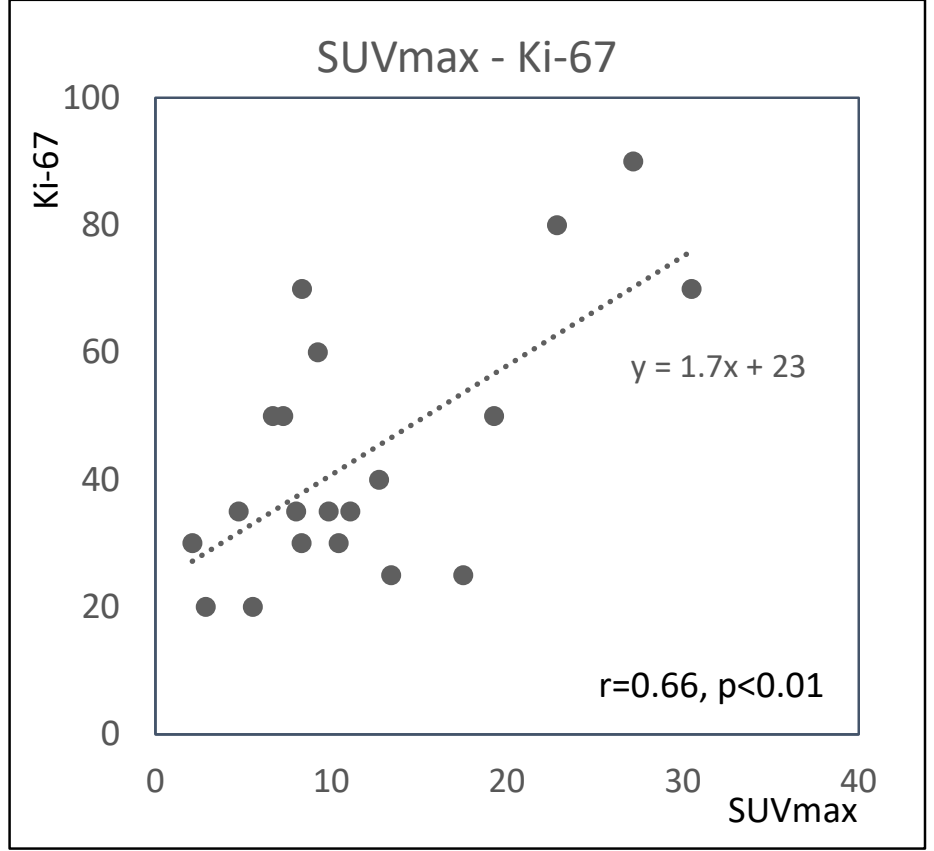

(c)

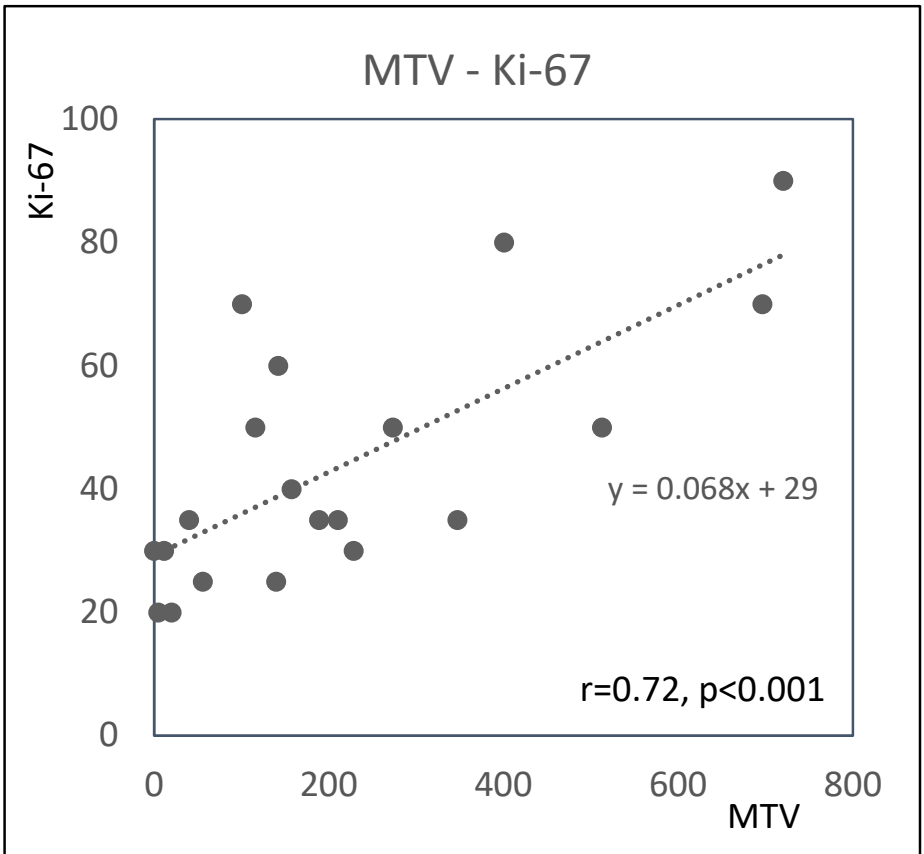

(b)

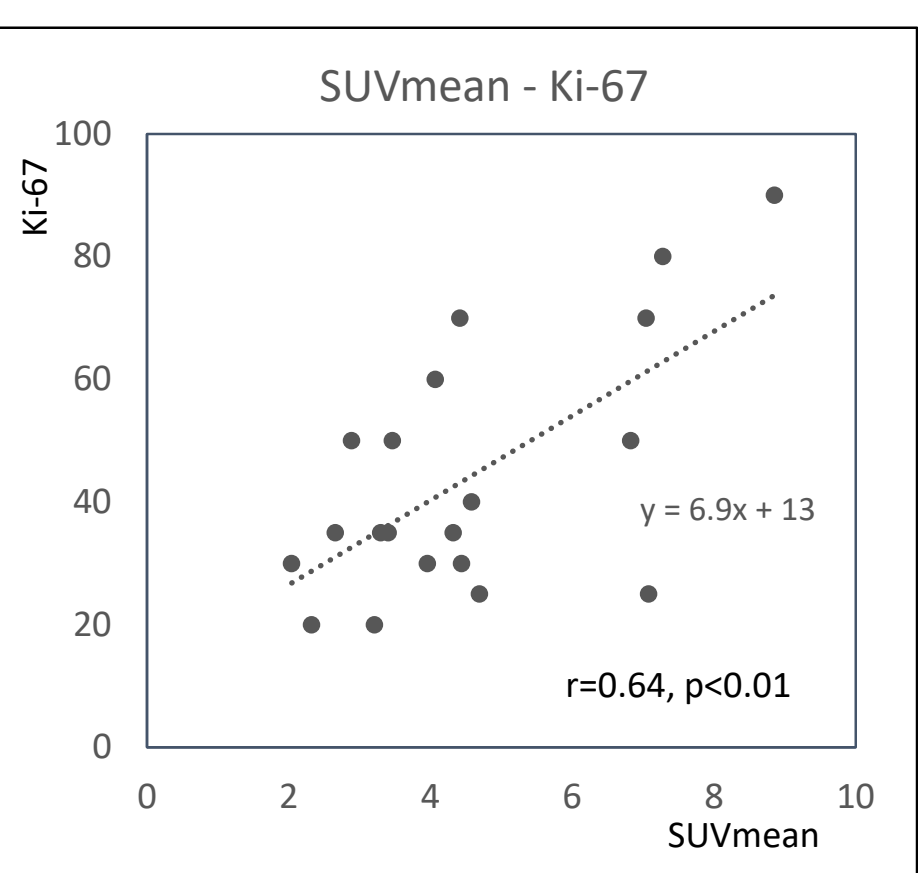

(d)

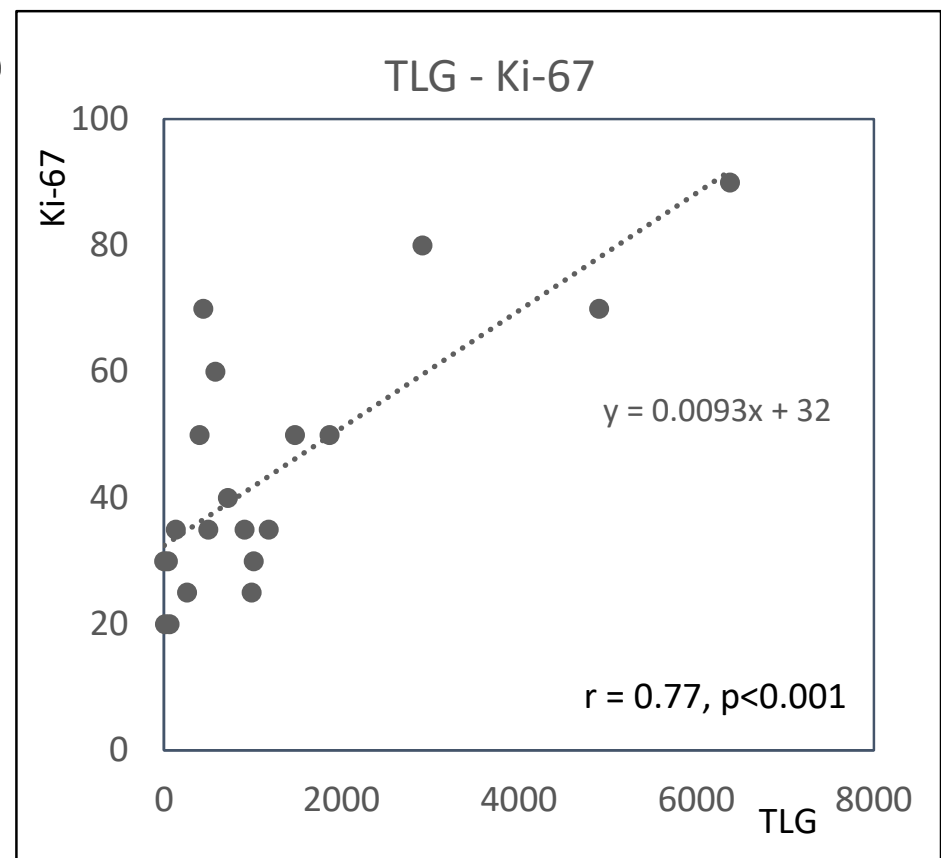


Fig. 4

(a)

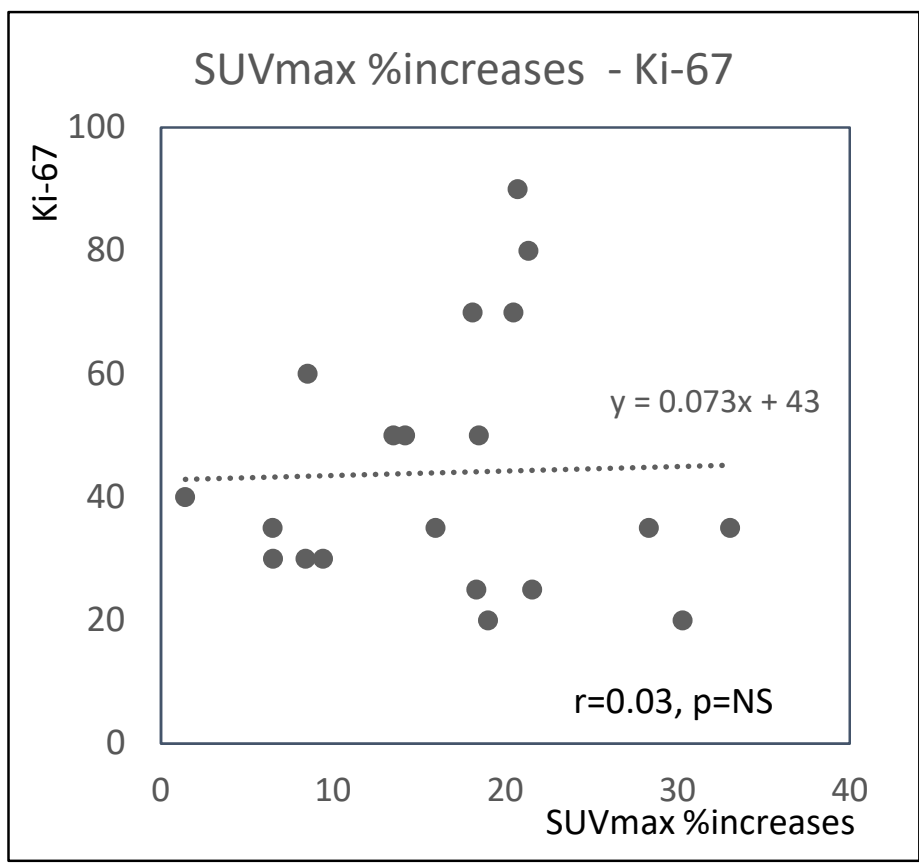

(c)

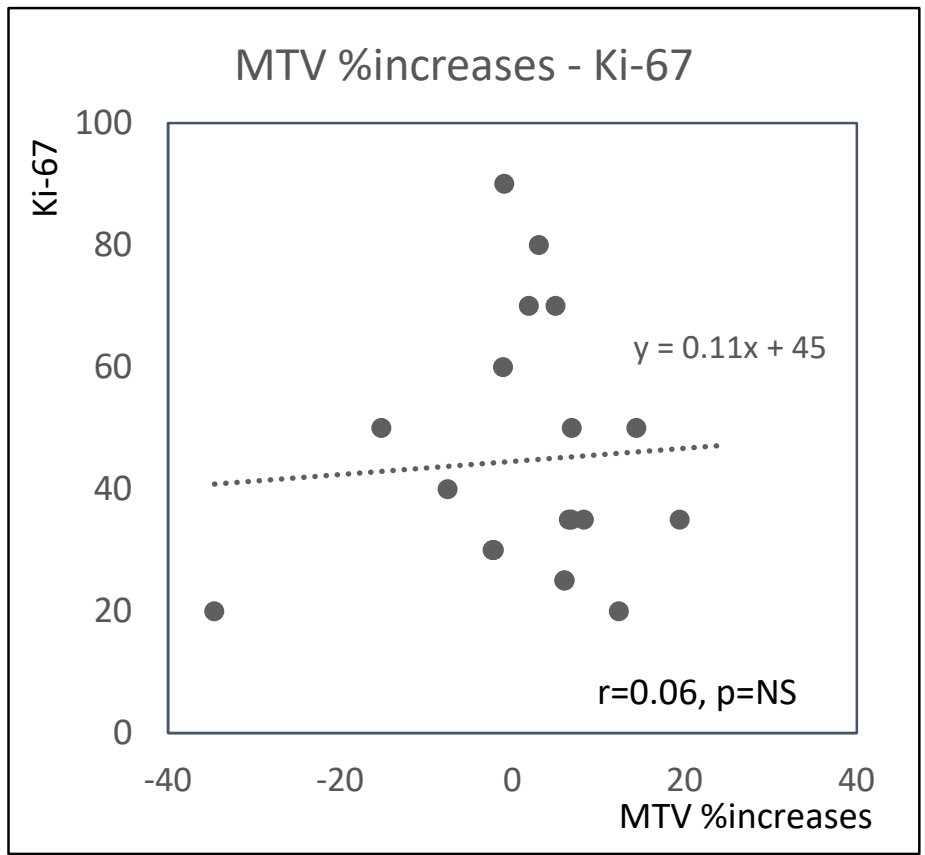

(b)

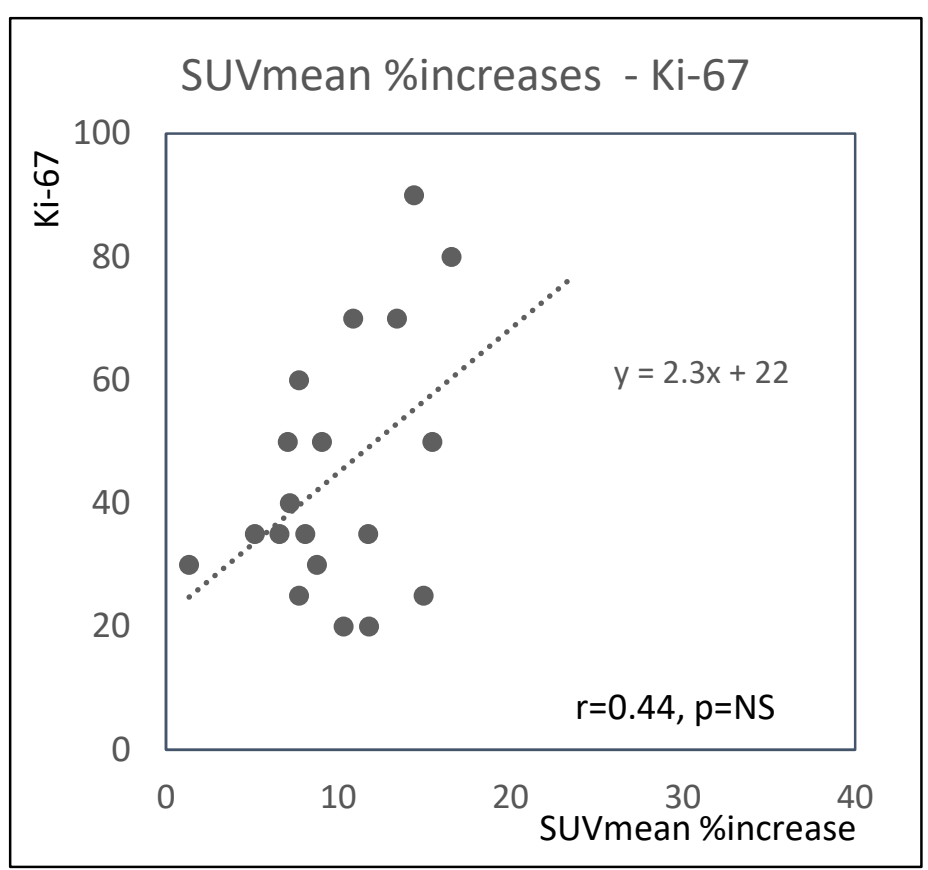

(d)

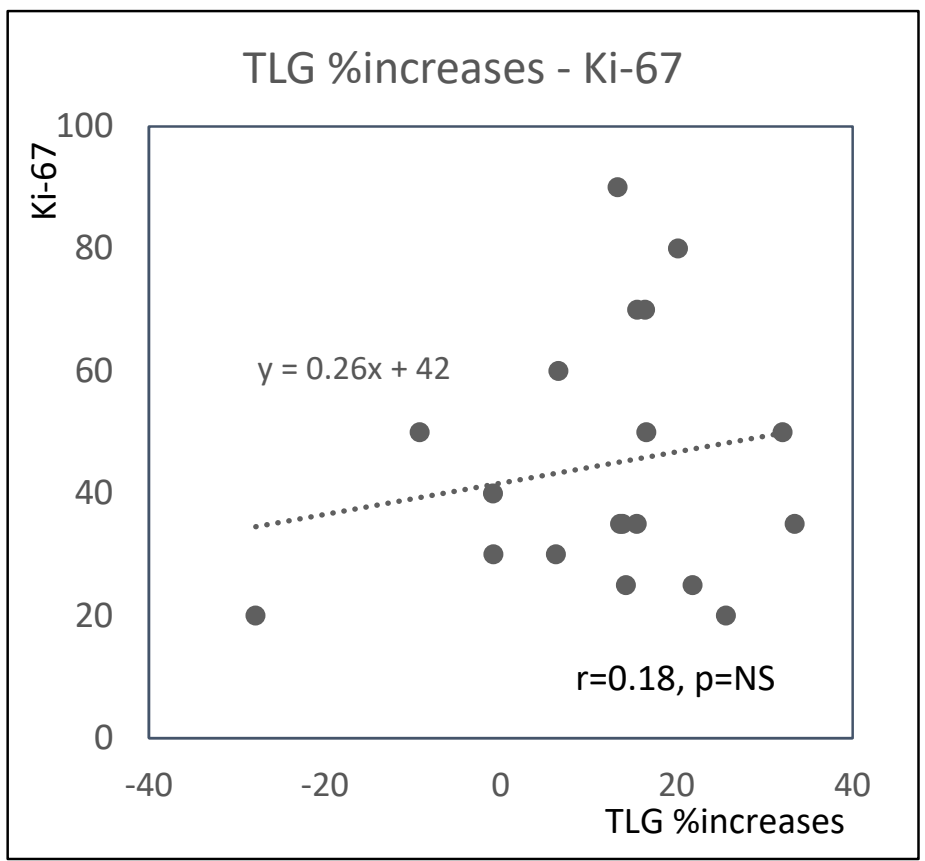


Fig. 5

(a)

(b)
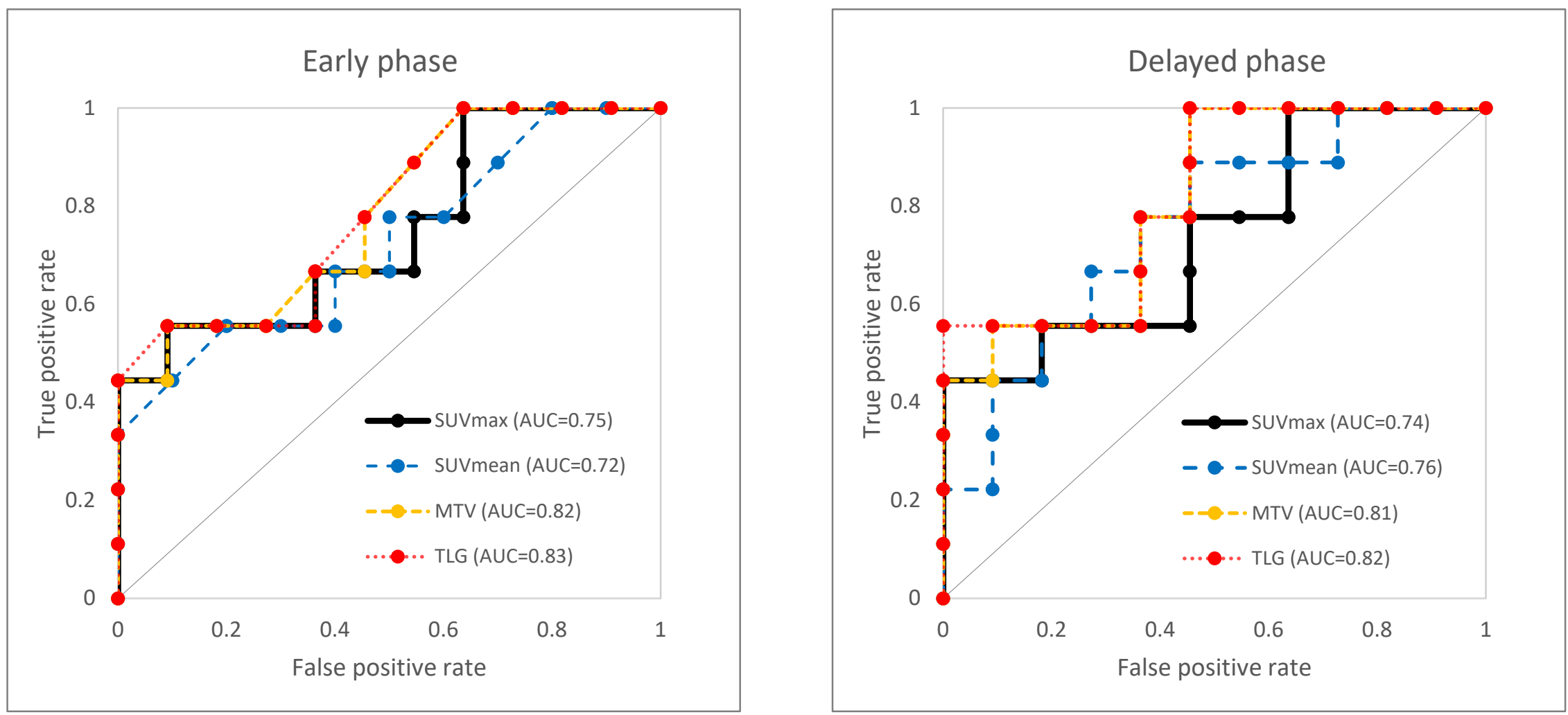
Fig. 6

SUV

0.0

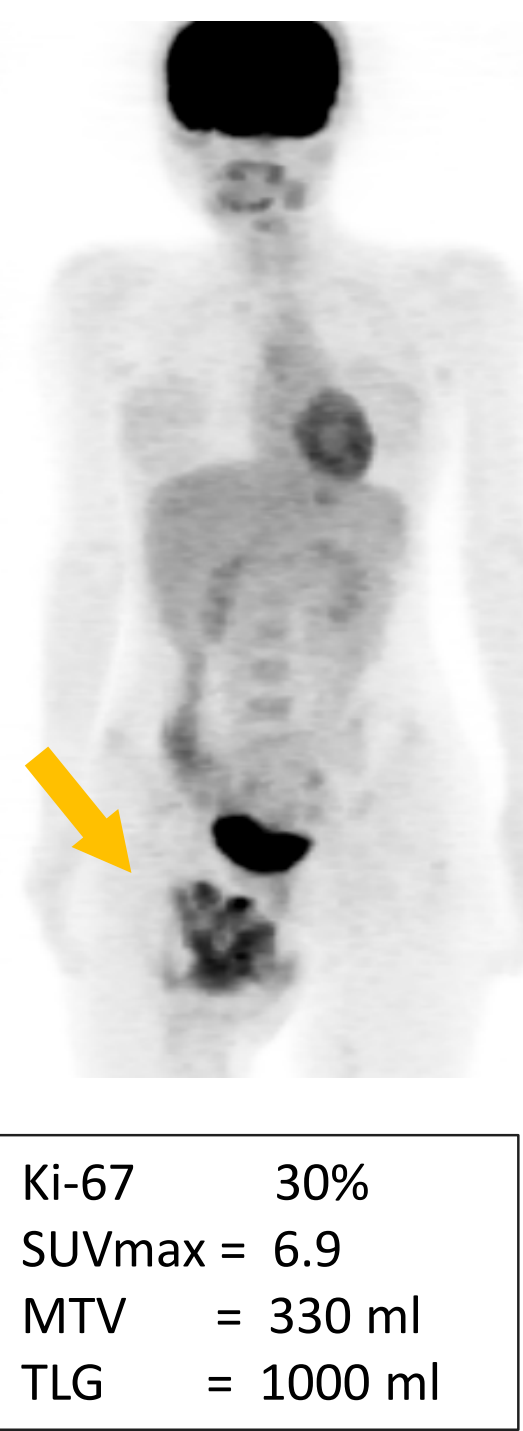

(a)

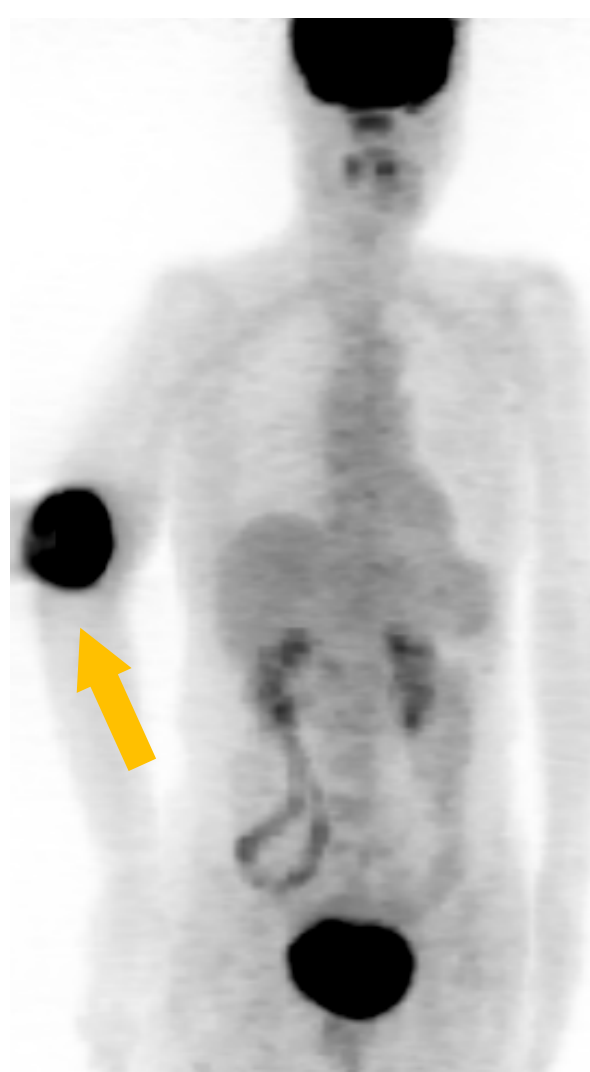

$\begin{array}{|ll|}\text { Ki-67 } & 50 \% \\ \text { SUVmax } & =17.0 \\ \text { MTV } & =240 \mathrm{ml} \\ \text { TLG } & =1400 \mathrm{ml}\end{array}$

(b)

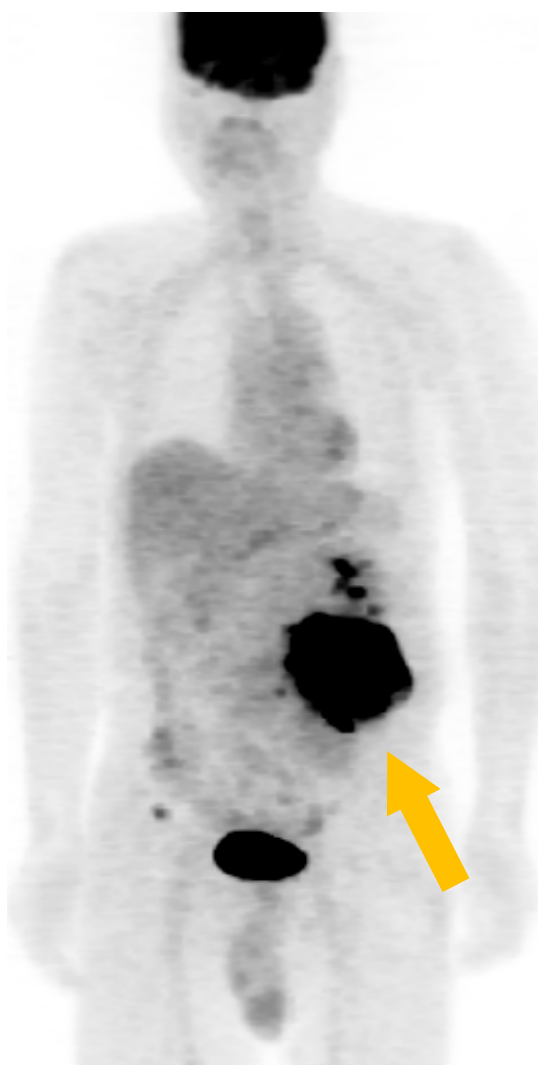

$\begin{array}{ll}\text { Ki-67 } & 90 \% \\ \text { SUVmax } & =23.0 \\ \text { MTV } & =730 \mathrm{ml} \\ \text { TLG } & =5600 \mathrm{ml}\end{array}$

(c) 